\title{
A Coloring Problem for Sturmian and Episturmian Words
}

\author{
Aldo de Luca ${ }^{1}$, Elena V. Pribavkina ${ }^{2}$, and Luca Q. Zamboni ${ }^{3}$ \\ 1 Dipartimento di Matematica \\ Università di Napoli Federico II, Italy \\ aldo.deluca@unina.it \\ 2 Ural Federal University, Ekaterinburg, Russia \\ elena.pribavkina@usu.ru \\ 3 Université Claude Bernard Lyon 1, France \\ and University of Turku, Finland \\ lupastis@gmail.com
}

\begin{abstract}
We consider the following open question in the spirit of Ramsey theory: Given an aperiodic infinite word $w$, does there exist a finite coloring of its factors such that no factorization of $w$ is monochromatic? We show that such a coloring always exists whenever $w$ is a Sturmian word or a standard episturmian word.
\end{abstract}

\section{Introduction}

Ramsey theory (including Van der Waerden's theorem) (see [5]) is a topic of great interest in combinatorics with connections to various fields of mathematics. A remarkable consequence of Ramsey's Infinitary Theorem applied to combinatorics on words yields the following unavoidable regularity of infinite words ${ }^{1}$ :

Theorem 1. Let $A$ be a non-empty alphabet, $w$ be an infinite word over $A, C$ a finite non-empty set (the set of colors), and $c$ : Fact ${ }^{+} w \rightarrow C$ any coloring of the set Fact $^{+} w$ of all non-empty factors of $w$. Then there exists a factorization of $w$ of the form $w=V U_{1} U_{2} \cdots U_{n} \cdots$ such that for all positive integers $i$ and $j, c\left(U_{i}\right)=c\left(U_{j}\right)$.

One can ask whether given an infinite word there exists a suitable coloring map able to avoid the monochromaticity of all factors in all factorizations of the word. More precisely, the following variant of Theorem 1 was posed as a question by T.C. Brown [3] and, independently, by the third author [11]:

Question 1 . Let $w$ be an aperiodic infinite word over a finite alphabet A. Does there exist a finite coloring $c:$ Fact $^{+} w \rightarrow C$ with the property that for any factoring $w=U_{1} U_{2} \cdots U_{n} \cdots$, there exist positive integers $i, j$ for which $c\left(U_{i}\right) \neq$ $c\left(U_{j}\right)$ ?

\footnotetext{
${ }^{1}$ Actually, the proof of Theorem 1 given by Schützenberger in [10] does not use Ramsey's theorem
} 
Let us observe that for periodic words the answer to the preceding question is trivially negative. Indeed, let $w=U^{\omega}$, and $c:$ Fact $^{+} w \rightarrow C$ be any finite coloring. By factoring $w$ as $w=U_{1} U_{2} \cdots U_{n} \cdots$, where for all $i \geq 1, U_{i}=U$ one has $c\left(U_{i}\right)=c\left(U_{j}\right)$ for all positive integers $i$ and $j$. It is easy to see that there exist non recurrent infinite words $w$ and finite colorings such that for any factoring $w=U_{1} U_{2} \cdots U_{n} \cdots$ there exist $i \neq j$ for which $c\left(U_{i}\right) \neq c\left(U_{j}\right)$. For instance, consider the infinite word $w=a b^{\omega}$ and define the coloring map as follows: for any non-empty factor $U$ of $w, c(U)=1$ if it contains $a$ and $c(U)=0$, otherwise. Then for any factoring $w=U_{1} U_{2} \cdots U_{n} \cdots, c\left(U_{1}\right)=1$ and $c\left(U_{i}\right)=0$ for all $i>1$.

It is not very difficult to prove that there exist infinite recurrent words for which Question 1 has a positive answer, for instance square-free, overlap-free words, and standard Sturmian words [11].

In this paper we show that Question 1 has a positive answer for every Sturmian word where the number of colors is equal to 3 . This solves a problem raised in [3] and in [11]. The proof requires some noteworthy new combinatorial properties of Sturmian words. Moreover, we prove that the same result holds true for aperiodic standard episturmian words by using a number of colors equal to the number of distinct letters occurring in the word plus one.

For all definitions and notation not explicitly given in the paper, the reader is referred to the books of M. Lothaire [7, 8]; for Sturmian words see [8, Chap. 2] and for episturmian words see $[4,6]$ and the survey of J. Berstel [1].

\section{Sturmian words}

There exist several equivalent definitions of Sturmian words. In particular, we recall (see, for instance, Theorem 2.1.5 of [8]) that an infinite word $s \in\{a, b\}^{\omega}$ is Sturmian if and only if it is aperiodic and balanced, i.e., for all factors $u$ and $v$ of $s$ such that $|u|=|v|$ one has:

$$
\left.|| u\right|_{x}-|v|_{x} \mid \leq 1, x \in\{a, b\},
$$

where $|u|_{x}$ denotes the number of occurrences of the letter $x$ in $u$. Since a Sturmian word $s$ is aperiodic, it must have at least one of the two factors $a a$ and $b b$. However, from the balance property, it follows that a Sturmian word cannot have both the factors $a a$ and $b b$.

Definition 1. We say that a Sturmian word is of type a (resp. b) if it does not contain the factor bb (resp. aa).

We recall that a factor $u$ of a finite or infinite word $w$ over the alphabet $A$ is called right special (resp. left special) if there exist two different letters $x, y \in A$ such that $u x, u y$ (resp. $x u, y u$ ) are factors of $w$.

A different equivalent definition of a Sturmian word is the following: A binary infinite word $s$ is Sturmian if for every integer $n \geq 0, s$ has a unique left (or equivalently right) special factor of length $n$. It follows from this that $s$ is closed under reversal, i.e., if $u$ is a factor of $s$ so is its reversal $u^{\sim}$. 
A Sturmian word $s$ is called standard (or characteristic) if all its prefixes are left special factors of $s$. As is well known, for any Sturmian word $s$ there exists a standard Sturmian word $t$ such that Fact $s=$ Fact $t$, where for any finite or infinite word $w$, Fact $w$ denotes the set of all its factors including the empty word.

Definition 2. Let $s \in\{a, b\}^{\omega}$ be a Sturmian word. A non-empty factor $w$ of $s$ is rich in the letter $z \in\{a, b\}$ if there exists a factor $v$ of $s$ such that $|v|=|w|$ and $|w|_{z}>|v|_{z}$.

From the aperiodicity and the balance property of a Sturmian word one easily derives that any non-empty factor $w$ of a Sturmian word $s$ is rich either in the letter $a$ or in the letter $b$ but not in both letters. Thus one can introduce for any given Sturmian word $s$ a map

$$
r_{s}: \text { Fact }^{+} s \rightarrow\{a, b\}
$$

defined as: for any non-empty factor $w$ of $s, r_{s}(w)=z \in\{a, b\}$ if $w$ is rich in the letter $z$. Clearly, $r_{s}(w)=r_{s}\left(w^{\sim}\right)$ for any $w \in$ Fact $^{+} s$.

For any letter $z \in\{a, b\}$ we shall denote by $\bar{z}$ the complementary letter of $z$, i.e., $\bar{a}=b$ and $\bar{b}=a$.

Lemma 1. Let $w$ be a non-empty right special (resp. left special) factor of a Sturmian word s. Then $r_{s}(w)$ is equal to the first letter of $w\left(r e s p . r_{s}(w)\right.$ is equal to the last letter of $w$ ).

Proof. Write $w=z w^{\prime}$ with $z \in\{a, b\}$ and $w^{\prime} \in\{a, b\}^{*}$. Since $w$ is a right special factor of $s$ one has that $v=w^{\prime} \bar{z}$ is a factor of $s$. Thus $|w|=|v|$ and $|w|_{z}>|v|_{z}$, whence $r_{s}(w)=z$. Similarly, if $w$ is left special one deduces that $r_{s}(w)$ is equal to the last letter of $w$.

\section{Preliminary Lemmas}

Lemma 2. Let $s$ be a Sturmian word such that

$$
s=\prod_{i \geq 1} U_{i}
$$

where the $U_{i}$ 's are non-empty factors of $s$. If for every $i$ and $j, r_{s}\left(U_{i}\right)=r_{s}\left(U_{j}\right)$, then for any $M>0$ there exists an integer $i$ such that $\left|U_{i}\right|>M$.

Proof. Suppose to the contrary that for some positive integer $M$ we have that $\left|U_{i}\right| \leq M$ for each $i \geq 1$. This implies that the number of distinct $U_{i}$ 's in the sequence $\left(U_{i}\right)_{i \geq 1}$ is finite, say $t$. Let $r_{s}\left(U_{i}\right)=x \in\{a, b\}$ for all $i \geq 1$ and set for each $i \geq 1$ :

$$
f_{i}=\frac{\left|U_{i}\right|_{x}}{\left|U_{i}\right|}
$$


Thus $\left\{f_{i} \mid i \geq 1\right\}$ is a finite set of at most $t$ rational numbers. We set $r=$ $\min \left\{f_{i} \mid i \geq 1\right\}$.

Let $f_{x}(s)$ be the frequency of the letter $x$ in $s$ defined as

$$
f_{x}(s)=\lim _{n \rightarrow \infty} \frac{\left|s_{[n]}\right| x}{n}
$$

where for every $n \geq 1, s_{[n]}$ denotes the prefix of $s$ of length $n$. As is well known (see Prop. 2.1.11 of [8]), $f_{x}(s)$ exists and is an irrational number.

Let us now prove that $r>f_{x}(s)$. From Proposition 2.1.10 in [8] one derives that for all $V \in$ Fact $s$

$$
|V| f_{x}(s)-1<|V|_{x}<|V| f_{x}(s)+1 .
$$

Now for any $i \geq 1, U_{i}$ is rich in the letter $x$, so that there exists $V_{i} \in$ Fact $s$ such that $\left|U_{i}\right|=\left|V_{i}\right|$ and $\left|U_{i}\right|_{x}>\left|V_{i}\right|_{x}$. From the preceding inequality one has:

$$
\left|U_{i}\right|_{x}=\left|V_{i}\right|_{x}+1>\left|V_{i}\right| f_{x}(s)=\left|U_{i}\right| f_{x}(s),
$$

so that for all $i \geq 1, f_{i}>f_{x}(s)$, hence $r>f_{x}(s)$.

For any $n>0$, we can write the prefix $s_{[n]}$ of length $n$ as:

$$
s_{[n]}=U_{1} \cdots U_{k} U_{k+1}^{\prime}
$$

for a suitable $k \geq 0$ and $U_{k+1}^{\prime}$ a prefix of $U_{k+1}$. Thus

$$
\left|s_{[n]}\right|_{x}=\sum_{i=i}^{k}\left|U_{i}\right|_{x}+\left|U_{k+1}^{\prime}\right|_{x} .
$$

Since $\left|U_{i}\right|_{x}=f_{i}\left|U_{i}\right| \geq r\left|U_{i}\right|$ and $\left|U_{k+1}^{\prime}\right| \leq M$, one has

$$
\left|s_{[n]}\right|_{x} \geq r \sum_{i=1}^{k}\left|U_{i}\right|=r\left(n-\left|U_{k+1}^{\prime}\right|\right) \geq r n-r M .
$$

Thus

$$
\frac{\left|s_{[n]}\right| x}{n} \geq r-r \frac{M}{n}
$$

and

$$
f_{x}(s)=\lim _{n \rightarrow \infty} \frac{\left|s_{[n]}\right|_{x}}{n} \geq r
$$

a contradiction.

In the following we shall consider the Sturmian morphism $R_{a}$, that we simply denote $R$, defined as:

$$
R(a)=a \text { and } R(b)=b a .
$$

For any finite or infinite word $w$, Pref $w$ will denote the set of all its prefixes. The following holds: 
Lemma 3. Let $s$ be a Sturmian word and $t \in\{a, b\}^{\omega}$ such that $R(t)=s$. If either

1) the first letter of $t$ (or, equivalently, of $s$ ) is $b$

or

2) the Sturmian word $s$ admits a factorization:

$$
s=U_{1} \cdots U_{n} \cdots,
$$

where each $U_{i}, i \geq 1$, is a non-empty prefix of $s$ terminating in the letter a and $r_{s}\left(U_{i}\right)=r_{s}\left(U_{j}\right)$ for all $i, j \geq 1$,

then $t$ is also Sturmian.

Proof. Let us prove that in both cases $t$ is balanced. Suppose to the contrary that $t$ is unbalanced. Then (see Prop. 2.1.3 of [8]) there would exists $v$ such that

$$
a v a, b v b \in \text { Fact } t \text {. }
$$

Thus

$$
a R(v) a, b a R(v) b a \in \text { Fact } s .
$$

If ava $\notin$ Pref $t$, then $t=\lambda a v a \mu$, with $\lambda \in\{a, b\}^{+}$and $\mu \in\{a, b\}^{\omega}$. Therefore $R(t)=R(\lambda) R($ ava $) R(\mu)$. Since the last letter of $R(\lambda)$ is $a$, it follows that $a a R(v) a \in$ Fact $s$. As $b a R(v) b \in$ Fact $s$ we reach a contradiction with the balance property of $s$. In case 1), $t$ begins in the letter $b$, so that ava $\notin$ Pref $t$ and then $t$ is balanced. In case 2) suppose that ava $\in$ Pref $t$. This implies that $a R(v) a \in$ Pref $s$. From the preceding lemma in the factorization of $s$ in prefixes there exists an integer $i>1$ such that $\left|U_{i}\right|>|a R(v) a|$. Since $U_{i-1}$ terminates in $a$ and $U_{i-1} U_{i} \in$ Fact $s$, it follows that $a a R(v) a \in$ Fact $s$ and one contradicts again the balance property of $s$. Hence, $t$ is balanced.

Trivially, in both cases $t$ is aperiodic, so that $t$ is Sturmian.

Let us remark that, in general, without any additional hypothesis, if $s=R(t)$, then $t$ need not be Sturmian. For instance, if $f$ is the Fibonacci word $f=$ abaababaaba $\cdots$, then af is also a Sturmian word. However, it is readily verified that in this case the word $t$ such that $R(t)=s$ is not balanced, so that $t$ is not Sturmian.

For any finite or infinite word $w$ over the alphabet $A$, alph $w$ denotes the set of all distinct letters of $A$ occurring in $w$. We will make use of the following lemma:

Lemma 4. Let $s$ be a Sturmian word having a factorization

$$
s=U_{1} \cdots U_{n} \cdots,
$$

where for $i \geq 1, U_{i}$ are non-empty prefixes of $s$. Then for any $p \geq 1, U_{1} \neq c^{p}$ where $c$ is the first letter of $s$.

Proof. Suppose that $U_{1}=c^{p}$. Since $s$ is aperiodic there exists a minimal integer $j$ such that $\operatorname{card}\left(\operatorname{alph} U_{j}\right)=2$. Since $U_{j}$ is a prefix of $s$, one has then $U_{1} \cdots U_{j-1} U_{j}=U_{j} \xi$, with $\xi \in\{a, b\}^{*}$. As $U_{1} \cdots U_{j-1}=c^{q}$ for a suitable $q \geq p$, it follows that $\xi=c^{q}$ and $U_{j} \in c c^{*}$, a contradiction. 


\section{Main results}

Proposition 1. Let s be a Sturmian word of type a having a factorization

$$
s=U_{1} \cdots U_{n} \cdots,
$$

where for $i \geq 1, U_{i}$ are non-empty prefixes of $s$ such that $r_{s}\left(U_{i}\right)=r_{s}\left(U_{j}\right)$ for all $i, j \geq 1$. Then one of the following two properties holds:

i) All $U_{i}, i \geq 1$, terminate in the letter a.

ii) For all $i \geq 1, U_{i} a \in \operatorname{Pref} s$.

Proof. Let us first suppose that $s$ begins in the letter $b$. All prefixes $U_{i}, i \geq 1$, of $s$ begin in the letter $b$ and, as $s$ is of type $a$, have to terminate in the letter $a$. Thus in this case Property $i$ ) is satisfied.

Let us then suppose that $s$ begins in the letter $a$. Now either all prefixes $U_{i}$, $i \geq 1$, terminate in the letter $a$ or all prefixes $U_{i}, i \geq 1$, terminate in the letter $b$ or some of the prefixes terminate in the letter $a$ and some in the letter $b$. We have then to consider the following cases:

Case 1. All prefixes $U_{i}, i \geq 1$, terminate in the letter $b$.

Since $s$ is of type $a$, no one of the prefixes $U_{i}, i \geq 1$, can be a right special factor. This implies that $U_{i} a \in \operatorname{Pref} s$ and Property $i i$ ) is satisfied.

Case 2. Some of the prefixes $U_{i}, i \geq 1$, terminate in the letter $a$ and some in the letter $b$.

We have to consider two subcases:

a). $r_{s}\left(U_{i}\right)=b$, for all $i \geq 1$.

As all $U_{i}, i \geq 1$, begin in $a$, if any $U_{i}$ were right special, then by Lemma 1 , $r_{s}\left(U_{i}\right)=a$, a contradiction. It follows that for all $i \geq 1, U_{i} a \in$ Pref $s$.

b). $r_{s}\left(U_{i}\right)=a$, for all $i \geq 1$.

Some of the prefixes $U_{j}, j \geq 1$, terminate in $a$ (since otherwise we are in Case 1). Let $U_{k}$ be a prefix terminating in $a$ for a suitable $k \geq 1$. If a prefix $U_{i}$ terminates in $b$, then $a U_{i}$ is not a factor of $s$. Indeed, otherwise, the word $a U_{i} b^{-1}$ is such that $\left|a U_{i} b^{-1}\right|=\left|U_{i}\right|$ and $\left|a U_{i} b^{-1}\right|_{b}<\left|U_{i}\right|_{b}$, so that $r_{s}\left(U_{i}\right)=b$ a contradiction. Thus one derives that all $U_{l}$ with $l \geq k$ terminate in $a$. Moreover, if some $U_{i}$ terminate in $b$, by Lemma 2 there exists $j>k$ such that $U_{j}$ has the prefix $U_{i}$, so that $U_{j-1} U_{i} \in$ Fact $s$. Since $U_{j-1}$ terminates in $a$, one has that $a U_{i}$ is a factor of $s$, a contradiction. Thus all $U_{i}, i \geq 1$, terminate in $a$.

Proposition 2. Let $s$ be a Sturmian word having a factorization

$$
s=U_{1} \cdots U_{n} \cdots,
$$

where for $i \geq 1, U_{i}$ are non-empty prefixes of $s$ such that $r_{s}\left(U_{i}\right)=r_{s}\left(U_{j}\right)$ for all $i, j \geq 1$. Then there exists a Sturmian word $t$ such that

$$
t=V_{1} \cdots V_{n} \cdots,
$$

where for all $i \geq 1, V_{i}$ are non-empty prefixes of $t, r_{t}\left(V_{i}\right)=r_{t}\left(V_{j}\right)$ for all $i, j \geq 1$, and $\left|V_{1}\right|<\left|U_{1}\right|$. 
Proof. We can suppose without loss of generality that $s$ is a Sturmian word of type $a$. From Proposition 1 either all $U_{i}, i \geq 1$, terminate in the letter $a$ or for all $i \geq 1, U_{i} a \in \operatorname{Pref} s$. We consider two cases:

Case 1. For all $i \geq 1, U_{i} a \in$ Pref $s$.

We can suppose that $s$ begins in the letter $a$. Indeed, otherwise, if the first letter of $s$ is $b$, then all $U_{i}, i \geq 1$, begin in the letter $b$ and, as $s$ is of type $a$, they have to terminate in the letter $a$. Thus the case that the first letter of $s$ is $b$ will be considered when we will analyze Case 2 .

We consider the injective endomorphism of $\{a, b\}^{*}, L_{a}$, or simply $L$, defined by

$$
L(a)=a \text { and } L(b)=a b .
$$

Since $s$ is of type $a$, the first letter of $s$ is $a$, and $X=\{a, a b\}$ is a code having a finite deciphering delay (cf. [2]), the word $s$ can be uniquely factorized by the elements of $X$. Thus there exists a unique word $t \in\{a, b\}^{\omega}$ such that $s=L(t)$. The following holds:

1. The word $t$ is a Sturmian word.

2. For any $i \geq 1$ there exists a non-empty prefix $V_{i}$ of $t$ such that $L\left(V_{i}\right)=U_{i}$.

3 . The word $t$ can be factorized as $t=V_{1} \cdots V_{n} \cdots$.

4. $\left|V_{1}\right|<\left|U_{1}\right|$.

5. For all $i, j \geq 1, r_{t}\left(V_{i}\right)=r_{t}\left(V_{j}\right)$.

Point 1 . This is a consequence of the fact that $L$ is a standard Sturmian morphism (see Corollary 2.3.3 in Chap. 2 of [8]).

Point 2. For any $i \geq 1$, since $U_{i} a \in \operatorname{Pref} s$ and any pair $(c, a)$ with $c \in\{a, b\}$ is synchronizing for $X^{\infty}=X^{*} \cup X^{\omega}$ (cf. [2]), one has that $U_{i} \in X^{*}$, so that there exists $V_{i} \in \operatorname{Pref} t$ such that $L\left(V_{i}\right)=U_{i}$.

Point 3. One has $L\left(V_{1} \cdots V_{n} \cdots\right)=U_{1} \cdots U_{n} \cdots=s=L(t)$. Thus $t=V_{1} \cdots V_{n} \cdots$.

Point 4. By Lemma 4, $U_{1}$ is not a power of $a$ so that in $U_{1}$ there must be at least one occurrence of the letter $b$. This implies that $\left|V_{1}\right|<\left|U_{1}\right|$.

Point 5. We shall prove that for all $i \geq 1, r_{t}\left(V_{i}\right)=r_{s}\left(U_{i}\right)$. From this one has that for all $i, j \geq 1, r_{t}\left(V_{i}\right)=r_{t}\left(V_{j}\right)$.

Since $t$ is a Sturmian word, there exists $V_{i}^{\prime} \in$ Fact $t$ such that

$$
\left|V_{i}\right|=\left|V_{i}^{\prime}\right| \text { and either }\left|V_{i}\right|_{a}>\left|V_{i}^{\prime}\right|_{a} \text { or }\left|V_{i}\right|_{a}<\left|V_{i}^{\prime}\right|_{a} .
$$

In the first case $r_{t}\left(V_{i}\right)=a$ and in the second case $r_{t}\left(V_{i}\right)=b$. Let us set

$$
F_{i}=L\left(V_{i}^{\prime}\right)
$$

Since $U_{i}=L\left(V_{i}\right)$, from the definition of the morphism $L$ one has:

$$
\begin{gathered}
\left|F_{i}\right|_{a}=\left|V_{i}^{\prime}\right|_{a}+\left|V_{i}^{\prime}\right|_{b}=\left|V_{i}^{\prime}\right|,\left|F_{i}\right|_{b}=\left|V_{i}^{\prime}\right|_{b} . \\
\left|U_{i}\right|_{a}=\left|V_{i}\right|_{a}+\left|V_{i}\right|_{b}=\left|V_{i}\right|,\left|U_{i}\right|_{b}=\left|V_{i}\right|_{b} .
\end{gathered}
$$


Let us first consider the case $r_{t}\left(V_{i}\right)=a$, i.e., $\left|V_{i}\right|_{a}=\left|V_{i}^{\prime}\right|_{a}+1$ and $\left|V_{i}\right|_{b}=$ $\left|V_{i}^{\prime}\right|_{b}-1$. From the preceding equations one has:

$$
\left|F_{i}\right|=\left|U_{i}\right|+1
$$

Moreover, from the definition of $L$ one has that $F_{i}$ begins in the letter $a$. Hence, $\left|a^{-1} F_{i}\right|=\left|U_{i}\right|$ and $\left|a^{-1} F_{i}\right|_{a}=\left|F_{i}\right|_{a}-1=\left|U_{i}\right|_{a}-1$. Thus $\left|U_{i}\right|_{a}>\left|a^{-1} F_{i}\right|_{a}$. Since $a^{-1} F_{i} \in$ Fact $s$, one has

$$
r_{s}\left(U_{i}\right)=r_{t}\left(V_{i}\right)=a .
$$

Let us now consider the case $r_{t}\left(V_{i}\right)=b$, i.e., $\left|V_{i}\right|_{a}=\left|V_{i}^{\prime}\right|_{a}-1$ and $\left|V_{i}\right|_{b}=\left|V_{i}^{\prime}\right|_{b}+1$. From (2) and (3) one derives:

$$
\left|U_{i}\right|=\left|F_{i}\right|+1,
$$

and $\left|U_{i}\right|_{b}>\left|F_{i}\right|_{b}$. Now $F_{i} a$ is a factor of $s$. Indeed, $F_{i}=L\left(V_{i}^{\prime}\right)$ and for any $c \in\{a, b\}$ such that $V_{i}^{\prime} c \in$ Fact $t$ one has $L\left(V_{i}^{\prime} c\right)=F_{i} L(c)$. Since for any letter $c, L(c)$ begins in the letter $a$ it follows that $F_{i} a \in$ Fact $s$. Since $\left|F_{i} a\right|=\left|U_{i}\right|$ and $\left|U_{i}\right|_{b}>\left|F_{i}\right|_{b}=\left|F_{i} a\right|_{b}$, one has that $U_{i}$ is rich in $b$. Hence, $r_{s}\left(U_{i}\right)=r_{t}\left(V_{i}\right)=b$.

Case 2. All $U_{i}, i \geq 1$, terminate in the letter $a$.

We consider the injective endomorphism of $\{a, b\}^{*}, R_{a}$, or simply $R$, defined in (1). Since $s$ is of type $a$ and $X=\{a, b a\}$ is a prefix code, the word $s$ can be uniquely factorized by the elements of $X$. Thus there exists a unique word $t \in\{a, b\}^{\omega}$ such that $s=R(t)$. The following holds:

1. The word $t$ is a Sturmian word.

2. For any $i \geq 1$ there exists a non-empty prefix $V_{i}$ of $t$ such that $R\left(V_{i}\right)=U_{i}$.

3 . The word $t$ can be factorized as $t=V_{1} \cdots V_{n} \cdots$.

4. $\left|V_{1}\right|<\left|U_{1}\right|$.

5. For all $i, j \geq 1, r_{t}\left(V_{i}\right)=r_{t}\left(V_{j}\right)$.

Point 1. From Lemma 3 , since $R(t)=s$ it follows that $t$ is Sturmian.

Point 2. For any $i \geq 1$, since $U_{i}$ terminates in the letter $a$ and any pair $(a, c)$ with $c \in\{a, b\}$ is synchronizing for $X^{\infty}$, one has that $U_{i} \in X^{*}$, so that there exists $V_{i} \in \operatorname{Pref} t$ such that $R\left(V_{i}\right)=U_{i}$.

Point 3. One has $R\left(V_{1} \cdots V_{n} \cdots\right)=U_{1} \cdots U_{n} \cdots=s=R(t)$. Thus $t=V_{1} \cdots V_{n} \cdots$.

Point 4. By Lemma $4, U_{1}$ is not a power of the first letter $c$ of $s$, so that in $U_{1}$ there must be at least one occurrence of the letter $\bar{c}$. This implies that $\left|V_{1}\right|<\left|U_{1}\right|$.

Point 5 . We shall prove that for all $i \geq 1, r_{t}\left(V_{i}\right)=r_{s}\left(U_{i}\right)$. From this one has that for all $i, j \geq 1, r_{t}\left(V_{i}\right)=r_{t}\left(V_{j}\right)$.

Since $t$ is a Sturmian word, there exists $V_{i}^{\prime} \in$ Fact $t$ such that

$$
\left|V_{i}\right|=\left|V_{i}^{\prime}\right| \text { and either }\left|V_{i}\right|_{a}>\left|V_{i}^{\prime}\right|_{a} \text { or }\left|V_{i}\right|_{a}<\left|V_{i}^{\prime}\right|_{a} .
$$

In the first case $r_{t}\left(V_{i}\right)=a$ and in the second case $r_{t}\left(V_{i}\right)=b$. Let us set

$$
F_{i}=R\left(V_{i}^{\prime}\right)
$$


Since $U_{i}=R\left(V_{i}\right)$, from the definition of the morphism $R$ one has that equations (2) and (3) are satisfied.

Let us first consider the case $r_{t}\left(V_{i}\right)=a$, i.e., $\left|V_{i}\right|_{a}=\left|V_{i}^{\prime}\right|_{a}+1$ and $\left|V_{i}\right|_{b}=$ $\left|V_{i}^{\prime}\right|_{b}-1$. From the preceding equations one has:

$$
\left|F_{i}\right|=\left|U_{i}\right|+1
$$

From the definition of the morphism $R$ one has that $F_{i}=R\left(V_{i}^{\prime}\right)$ terminates in the letter $a$. Hence, $\left|F_{i} a^{-1}\right|=\left|U_{i}\right|$ and $\left|F_{i} a^{-1}\right|_{a}=\left|F_{i}\right|_{a}-1=\left|U_{i}\right|_{a}-1$. Thus $\left|U_{i}\right|_{a}=\left|F_{i} a^{-1}\right|_{a}+1$, so that $U_{i}$ is rich in $a$ and $r_{s}\left(U_{i}\right)=r_{t}\left(V_{i}\right)=a$.

Let us now suppose that $r_{t}\left(V_{i}\right)=b$, i.e., $\left|V_{i}\right|_{a}=\left|V_{i}^{\prime}\right|_{a}-1$ and $\left|V_{i}\right|_{b}=\left|V_{i}^{\prime}\right|_{b}+1$. From (2) and (3) one derives:

$$
\left|U_{i}\right|=\left|F_{i}\right|+1
$$

and $\left|U_{i}\right|_{b}>\left|F_{i}\right|_{b}$. We prove that $a F_{i} \in$ Fact $s$. Indeed, $F_{i}=R\left(V_{i}^{\prime}\right)$ and for any $c \in\{a, b\}$ such that $c V_{i}^{\prime} \in$ Fact $t$ one has $R(c) R\left(V_{i}^{\prime}\right)=R(c) F_{i}$. Note that such a letter $c$ exists always as $t$ is recurrent. Since for any letter $c, R(c)$ terminates in the letter $a$ it follows that $a F_{i} \in$ Fact $s$. Since $\left|a F_{i}\right|=\left|U_{i}\right|$ and $\left|U_{i}\right|_{b}>\left|a F_{i}\right|_{b}=\left|F_{i}\right|_{b}$, one has that $U_{i}$ is rich in $b$. Hence, $r_{s}\left(U_{i}\right)=r_{t}\left(V_{i}\right)=b$.

Theorem 2. Let $s$ be a Sturmian word having a factorization

$$
s=U_{1} \cdots U_{n} \cdots,
$$

where each $U_{i}, i \geq 1$, is a non-empty prefix of $s$. Then there exist integers $i, j \geq 1$ such that $r_{s}\left(U_{i}\right) \neq r_{s}\left(U_{j}\right)$.

Proof. Let $s$ be a Sturmian word and suppose that $s$ admits a factorization

$$
s=U_{1} \cdots U_{n} \cdots,
$$

where for $i \geq 1, U_{i}$ are non-empty prefixes such that for all $i, j \geq 1, r_{s}\left(U_{i}\right)=$ $r_{s}\left(U_{j}\right)$. Among all Sturmian words having this property we can always consider a Sturmian word $s$ such that $\left|U_{1}\right|$ is minimal. Without loss of generality we can suppose that $s$ is of type $a$. By Proposition 2 there exists a Sturmian word $t$ such that

$$
t=V_{1} \cdots V_{n} \cdots,
$$

where for all $i \geq 1, V_{i}$ are non-empty prefixes, $r_{t}\left(V_{i}\right)=r_{t}\left(V_{j}\right)$ for all $i, j \geq 1$, and $\left|V_{1}\right|<\left|U_{1}\right|$, that contradicts the minimality of the length of $U_{1}$.

Theorem 3. Let $s$ be a Sturmian word. There exists a coloring $c$ of the nonempty factors of $s, c:$ Fact $^{+} s \rightarrow\{0,1,2\}$ such that for any factorization

$$
s=V_{1} \cdots V_{n} \cdots
$$

in non-empty factors $V_{i}, i \geq 1$, there exist integers $i, j$ such that $c\left(V_{i}\right) \neq c\left(V_{j}\right)$. 
Proof. Let us define the coloring $c$ as: for any $V \in \mathrm{Fact}^{+} s$

$$
c(V)=\left\{\begin{array}{l}
0 \text { if } V \text { is not a prefix of } s \\
1 \text { if } V \text { is a prefix of } s \text { and } r_{s}(V)=a \\
2 \text { if } V \text { is a prefix of } s \text { and } r_{s}(V)=b
\end{array}\right.
$$

Let us suppose to contrary that for all $i, j, c\left(V_{i}\right)=c\left(V_{j}\right)=x \in\{0,1,2\}$. If $x=0$ we reach a contradiction as $V_{1}$ is a prefix of $s$ so that $c\left(V_{1}\right) \in\{1,2\}$. If $x=1$ or $x=2$, then all $V_{i}$ have to be prefixes of $s$ having the same richness, but this contradicts Theorem 2.

\section{The case of standard episturmian words}

An infinite word $s$ over the alphabet $A$ is called standard episturmian if it is closed under reversal and every left special factor of $s$ is a prefix of $s$. A word $s \in A^{\omega}$ is called episturmian if there exists a standard episturmian $t \in A^{\omega}$ such that Fact $s=$ Fact $t$. We recall the following facts about episturmian words $[4$, $6]$ :

Fact 1. Every prefix of an aperiodic standard episturmian word $s$ is a left special factor of $s$. In particular an aperiodic standard episturmian word on a two-letter alphabet is a standard Sturmian word.

Fact 2. If $s$ is a standard episturmian word with first letter $a$, then $a$ is separating, i.e., for any $x, y \in A$ if $x y \in$ Fact $s$, then $a \in\{x, y\}$.

For each $x \in A$, let $L_{x}$ denote the standard episturmian morphism [6] defined for any $y \in A$ by $L_{x}(y)=x$ if $y=x$ and $L_{x}(y)=x y$ for $x \neq y$.

Fact 3 . The infinite word $s \in A^{\omega}$ is standard episturmian if and only if there exist a standard episturmian word $t$ and $a \in A$ such that $s=L_{a}(t)$. Moreover, $t$ is unique and the first letter of $s$ is $a$.

The following was proved in [9]:

Fact 4. A recurrent word $w$ over the alphabet $A$ is episturmian if and only if for each factor $u$ of $w$, a letter $b$ exists (depending on $u$ ) such that $A u A \cap$ Fact $w \subseteq$ bu $A \cup A u b$.

Definition 3. We say that a standard episturmian word $s$ is of type $a, a \in A$, if the first letter of $s$ is a.

Theorem 4. Let $s$ be an aperiodic standard episturmian word over the alphabet $A$ and let $s=U_{1} U_{2} \cdots$ be any factoring of $s$ with each $U_{i}, i \geq 1$, a non-empty prefix of $s$. Then there exist indices $i \neq j$ for which $U_{i}$ and $U_{j}$ terminate in a different letter.

Proof. Suppose to the contrary that there exists an aperiodic standard episturmian word $s$ over the alphabet $A$ admitting a factorization $s=U_{1} U_{2} \cdots$ in which all $U_{i}$ are non-empty prefixes of $s$ ending in the same letter. Amongst all 
aperiodic standard episturmian words over the alphabet $A$ having the preceding factorization, we may choose one such $s$ for which $\left|U_{1}\right|$ is minimal. Let $a \in A$ be the first letter of $s$, so that $s$ is of type $a$.

Let us now prove that for every $i \geq 0$, one has that $U_{i} a$ is a prefix of $s$. Let us first suppose that for all $i \geq 1, U_{i}$ ends in a letter $x \neq a$. Since $a$ is separating $(s$ is of type $a$ ), $x$ can be followed only by $a$, so that the prefix $U_{i}$ can be followed only by $a$. This implies that $U_{i} a$ is a prefix of $s$.

Let us then suppose that for all $i \geq 1, U_{i}$ ends in $a$. Since $U_{1}$ is a prefix of $s$, and all $U_{i}, i \geq 1$, begin in $a$ one has that $U_{1} a$ is a prefix of $s$. Now let $i>1$. Since $U_{i-1}$ ends in $a$ it follows that $a U_{i} a$ is a factor of $s$.

Let $U_{i} x$ be a prefix of $s$; we want to show that $x=a$. Since $U_{i} x$ is left special (as it is a prefix of $s$ ), there exists a letter $y \neq a$ such that $y U_{i} x$ is a factor of $s$. Now from this and by Fact 4 , there exists a letter $b$ (depending only on $U_{i}$ ) such that either $x=b$ or $y=b$.

So now, by Fact 4, since $a U_{i} a$ and $y U_{i} x$ are both factors of $s$, we deduce $b=a$ and either $x=a$ or $y=a$. Since $y \neq a$, it follows that $x=a$. Therefore, we have proved that for every $i \geq 1, U_{i} a$ is a prefix of $s$.

Let us now observe that $U_{1}$ must contain the occurrence of a letter $x \neq a$. Indeed, otherwise, suppose that $U_{1}=a^{k}$ and consider the least $i>1$ such that $x$ occurs in $U_{i}$. This implies, by using an argument similar to that of the proof of Lemma 4, that $U_{i}$ cannot be a prefix of $s$.

By Fact 3, one has that there exists a unique standard episturmian word $s^{\prime}$ such that $s=L_{a}\left(s^{\prime}\right)$ and alph $s^{\prime} \subseteq$ alph $s \subseteq A$. Moreover, since $s$ is aperiodic, trivially one has that also $s^{\prime}$ is aperiodic.

Let us observe that the set $X=\{a\} \cup\{a x \mid x \in A\}$ is a code having deciphering delay equal to 1 and that any pair $(x, a)$ with $x \in A$ is synchronizing for $X^{\infty}$. This implies that $s$ can be uniquely factored by the words of $X$. Moreover, since $U_{i} a$ is a prefix of $s$, from the synchronization property of $X^{\infty}$, it follows that for each $i \geq 1$,

$$
U_{i}=L_{a}\left(U_{i}^{\prime}\right),
$$

where $U_{i}^{\prime}$ is a prefix of $s^{\prime}$. From the definition of $L_{a}$ and the preceding formula, one has that the last letter of $U_{i}$ is equal to the last letter of $U_{i}^{\prime}$.

Moreover,

$$
L_{a}\left(U_{1}^{\prime} \cdots U_{n}^{\prime} \cdots\right)=U_{1} \cdots U_{n} \cdots=s=L_{a}\left(s^{\prime}\right) .
$$

Thus $s^{\prime}=U_{1}^{\prime} \cdots U_{n}^{\prime} \cdots$, where each $U_{i}^{\prime}, i \geq 1$, is a non-empty prefix of $s^{\prime}$ and for all $i, j \geq 1, U_{i}^{\prime}$ and $U_{j}^{\prime}$ terminate in the same letter. Since in $U_{1}=L_{a}\left(U_{1}^{\prime}\right)$ there is the occurrence of a letter different from $a$ one obtains that $\left|U_{1}^{\prime}\right|<\left|U_{1}\right|$ which is a contradiction.

Let us observe that in the case of a standard Sturmian word, Theorem 4 is an immediate consequence of Theorem 2 and Lemma 1.

Theorem 5. Let $s$ be an aperiodic standard episturmian word and let $k=$ card(alph $s)$. There exists a coloring $c$ of the non-empty factors of $s, c:$ Fact $^{+} s \rightarrow$ 
$\{0,1, \ldots, k\}$ such that for any factorization

$$
s=V_{1} \cdots V_{n} \cdots
$$

in non-empty factors $V_{i}, i \geq 1$, there exist integers $i, j$ such that $c\left(V_{i}\right) \neq c\left(V_{j}\right)$.

Proof. Let alph $s=\left\{a_{1}, \ldots, a_{k}\right\}$. We define the coloring $c$ as: for any $V \in$ Fact $^{+} s$

$$
c(V)=\left\{\begin{array}{l}
0 \text { if } V \text { is not a prefix of } s \\
1 \text { if } V \text { is a prefix of } s \text { terminating in } a_{1} \\
\vdots \vdots \\
k \text { if } V \text { is a prefix of } s \text { terminating in } a_{k}
\end{array}\right.
$$

Let us suppose by contradiction that for all $i, j, c\left(V_{i}\right)=c\left(V_{j}\right)=x \in\{0,1, \ldots, k\}$. If $x=0$ we reach a contradiction as $V_{1}$ is a prefix of $s$ so that $c\left(V_{1}\right) \in\{1, \ldots, k\}$. If $x \in\{1, \ldots, k\}$, then all $V_{i}$ have to be prefixes of $s$ terminating in the same letter, but this contradicts Theorem 4.

\section{Acknowledgments}

The authors are indebted to Tom Brown for his suggestions and comments. The second author acknowledges support from the Presidential Program for young researchers, grant MK-266.2012.1. The third author is partially supported by a FiDiPro grant from the Academy of Finland.

\section{References}

1. Berstel, J.: Sturmian and Episturmian words (A survey of some recent results). LNCS, vol. 4728, Springer, Berlin, 2007, pp. 23-47

2. Berstel, J., Perrin, D., Reutenauer, C.: Codes and Automata, Cambridge University Press, 2010

3. Brown, T.C.: Colorings of the factors of a word, preprint Department of Mathematics, Simon Fraser University, Canada (2006)

4. Droubay, X., Justin, J., Pirillo, G.: Episturmian words and some constructions of de Luca and Rauzy, Theoret. Comput. Sci. 255 (2001) 539-553

5. Graham, R., Rothshild, B.L., Spencer, J.H.: Ramsey Theory, J. Wiley, New York, 2-nd edition, 1990

6. Justin, J., Pirillo, G.: Episturmian words and episturmian morphisms, Theoret. Comput. Sci. 276 (2002) 281-313

7. Lothaire, M.: Combinatorics on Words, Addison-Wesley Reading, MA, 1983, reprinted by Cambridge University Press, Cambridge, 1997

8. Lothaire, M.: Algebraic Combinatorics on Words, Cambridge University Press, Cambridge, 2002

9. Richomme, G.: A local Balance Property of Episturmian words, LNCS, vol. 4588, Springer, Berlin, 2007, pp. 371-381

10. Schützenberger, M. P.: Quelques problèmes combinatoires de la théorie des automates, Cours professé à l'Institut de Programmation en 1966/67, notes by J.-F. Perrot

11. Zamboni, L. Q.: A Note on Coloring Factors of Words, in Oberwolfach Report 37/2010, Mini-workshop: Combinatorics on Words, August 22-27, 2010, pp. 42-44 